\title{
RESEARCH ETHICS
}

\section{Responsibilities and obligations of using human research specimens transported across national boundaries}

\author{
A S Muula, J M Mfutso-Bengo
}

J Med Ethics 2007;33:35-38. doi: 10.1136/jme.2005.012492

Research collaboration beyond national jurisdiction is one aspect of the globalisation of health research. It has potential to complement researchers in terms of research skills, equipment and lack of adequate numbers of potential research subjects. Collaboration at an equal level of partnership though desirable, may not be practicable. Sometimes, human research specimens must be transported from one country to other. Where this occurs, there should be clear understanding between the collaborating research institutions regarding issues of access and control of the specimens as well as the duration of storage of specimens. The researchers have the duty to inform the research participants about specimen storage and transport across national boundaries. While obtaining informed consent from study subjects if specimens are to be stored beyond the life of the present study could be the ideal, there still remains significant challenges in a multi-cultural world.

See end of article for authors' affiliations

Correspondence to A S Muula, Department of Community Health, University of Malawi, College of Medicine, Private Bag 360, Chichiri, Blantyre 3, Malawi; a_muula@ yahoo.com s nternational research collaboration may necessamples from one country to another. When an institution from a developing nation collaborates with one from the developed nation, the usual practice is for specimens collected in the developing country to be transported to, stored and analysed in the developed nations, and not the other way round. Those transporting human specimens must adhere to the regulations set by the International Air Transport Association, United Nations Committee of Experts on the Transport of Dangerous Goods, the Universal Postal Union and the World Health Organization (WHO). Individual countries have their own guidelines on the transport of biological materials on domestic routes.

As human specimens may be transported, stored and analysed in a country other than where they were collected, it is necessary to develop ethical guidelines for conduct regarding the duration of storage, the purpose for which the specimens are intended, informed consent, ownership and access issues. In this paper, we suggest what we perceive to be important concepts and principles that should be always considered when human research specimens are transported from one national jurisdiction to another. We are writing from the perspective of a developing nation, Malawi, which is more often the source of specimens than a recipient. sitate the transfer of human specimens or

\section{MEDICAL RESEARCH IN MALAWI}

Much of the research on human subjects in Malawi is in the discipline of infectious diseases, mostly tuberculosis, HIV and AIDS, other sexually transmitted infections and malaria. Research on non-communicable diseases such as diabetes, hypertension and cancer is almost non-existent.

Health research in Malawi is mostly funded through international resources. Major funding for research on collection of human specimens comes through the collaboration of national research training institutions and international (foreign) institutions. The Wellcome Trust (UK), Fogarty International, the Centers for Disease Control and Prevention and the Bill and Melinda Gates Foundation, the Global Fund, the Department for International Development (UK), Manerheim League (Finland) and the Embassy of The Netherlands in Zambia contribute significantly to research in Malawi. The contribution from local pharmaceutical industry is not significant.

\section{HEALTHCARE DELIVERY IN MALAWI}

Malawi is a southeastern African country, burdened with communicable diseases. Maternal mortality is estimated at 1200 deaths per 100000 live births, overall HIV infection rate at $11-14 \%$ of the population and an infant death rate at 104 deaths per 1000 live births. ${ }^{1-4}$

The public health sector is the largest provider of healthcare, contributing $67 \%$ of formal healthcare, followed by the not-for-profit mission hospitals (Christian Health Association), which provide $31 \%$. The private health sector is expanding, but is yet to make major contributions to healthcare with regard to the number of patients seen. The public health facilities and those of Christian Health Association are organised in a hierarchy of about 20 categories, with the major ones being central hospital, district, subdistrict, community hospital, health centre, dispensary and maternity unit. ${ }^{1}$ Virtually all study participants in health research associated with collecting human specimens are patients attending care in public health facilities. Although there are private sections within public district and central hospitals, the bulk of care is provided at no cost to the patient.

\section{ETHICS REVIEW OF RESEARCH PROTOCOLS}

The National Research Council (NRCM) is mandated by an Act of Parliament to be the overall overseer of all research activities in Malawi. The health sciences research subcommittee of the NRCM is responsible for the review of the protocol 
and data and monitoring the safety of health research. This subcommittee holds regular meetings four times a year. On realising that there were an unusual greater number of proposals, especially from the College of Medicine, than the committee could handle at any one meeting, permission was granted to the College of Medicine (COM) to form its own committee, the College of Medicine Research and Ethics Committee (COMREC). The COMREC reviews all proposals from the faculty and students of the COM and the Kamuzu College of Nursing, and institutions and researchers affiliated to these training institutions. The COMREC reports to the National Health Sciences Research Committee and the heads of department at the COM. The arrangement is that the National Health Sciences Committee will not review research proposals from the COM and its affiliates unless the COMREC has referred the proposal to the higher body. Research that is identified as being of "sensitive national importance", such as HIV vaccine trials, is reviewed by the national committee. Decisions of the committee are reached by consensus.

The COMREC has representatives from clinical and nonclinical departments at the COM, the Ministry of Health, the NRCM, the Kamuzu College of Nursing and a lay member. A permanent seat is always kept for a bioethicist (JMM-B). The National Health Sciences Committee comprises representatives from the Ministry of Health, the NRCM, the Kamuzu College of Nursing, the COM, a bioethicist (JMM-B) and a lay member. The level of training in ethics review varies among the members and is not a requirement for membership. Lately however, training in research ethics through Fogarty Bioethics Training grant for southern Africa, the Wellcome Trust and the COM. From 2001, the COM introduced mandatory bioethics training for all its undergraduate medical students in each of the 5 years of medical school.

\section{INFORMED CONSENT}

Obtaining informed consent for all research on human subjects is necessary for approval of research protocols in Malawi. As research protocols are presented in English, research committees also require a translated copy of the informed consent process and form in Chichewa and any other native language, if the study is to be conducted in an area where participants are not likely to be native Chichewa speakers. Chichewa is one of the national languages in Malawi, the other being English. Most Malawians speak Chichewa.

For the informed consent process to be meaningful to potential study participants, they should be provided with information about the study and potential harms and benefits; they should be able to understand what they are told and be allowed to ask questions; and there should be a choice of whether to participate or not. When the participants have accepted participation, they should also know that they are free to leave the study at any time, without being penalised or suffering any losses.

The literacy level (defined as the ability to read and write) is about $67 \%$ and $45 \%$ among adult Malawian men and women, respectively. ${ }^{5}$ In most health research, written consent is preferred but oral consent is acceptable especially whereliteracy rates are low. Most research protocols have elaborate guidelines on how to obtain consent. We are, however, unaware of how potential research subjects generally perceive the informed consent process in Malawi. This is currently a question under study in a large anthropological study in Malawi in the Department of Community Health, COM. Although the informed consent process is elaborate, it is not required by any of the ethics committees that participants be told what will happen to the specimens with regard to being transported, stored and analysed overseas. We believe this is an important omission in the informed consent process.

\section{INTENTION OF USE}

One of the crucial issues to consider is the purpose for which the collection of the specimen is intended. As a part of the research, specimens are collected to help in answering questions for which the proposed research is being undertaken. The research subject, therefore, are expected to give (informed) consent on the basis of what they understand to be the object and rationale of the research. Institutional review boards (IRBs) or research ethics committees therefore have the responsibility of ensuring that human specimens collected from one jurisdiction transported to another are subjected to only those analyses specified in the research protocol. To go beyond the specified, and already agreed, intent of use can be construed as unethical, as it violates the principle of autonomy. Some authors have criticised the individualistic approach to autonomy, suggesting that the Western idea of autonomy may be problematic. ${ }^{6}$

Now the question is what should be done when it is perceived that the specimens already collected for one study may be useful for further analyses? Several options are available. The first is to consider collecting new samples altogether and using these for the currently proposed study. The problem with this is that it may be expensive to repeat the exercise. Informed consent, however, can be obtained and therefore the use of the same samples may be justified. This may be relatively practical if permission is to be obtained from the ethics review board that approved the initial study. We are suggesting that IRBs in the developing and the developed countries should assess the request and grant or not grant permission for use of the samples beyond the purposes of the study for which they were collected. The third alternative is that the researchers should seek consent once more from the research subjects who submitted the specimen. Although this may serve the principles of autonomy of research subjects, the practicability is extremely difficult, especially if the specimens were collected anonymously.

\section{JUSTIFICATION FOR EXPORT}

When human samples are to be shipped from one national authority to another as part of a research study, there should be justification for such export. In Malawi, most of the transported human specimens are sent out for DNA studies, although other specimens for relatively simple tests may also be sent abroad. No guidance exists on which tests should be or may be conducted abroad. The lack of adequate expertise and infrastructure is cited as a reason for such a practice. The export of samples may be justified if there was a lack of expertise or equipment. IRBs should, however, be able to assess whether any effort is being made to capacitate the "weaker" partner through training and equipment supplies. The duration of the study may also be considered to assess whether the export of samples is justified or not. For instance, if a research project has possibilities of operating in an area for more than 2 years, it may be recommended that capacity building through transfer of equipment should form part of the funding. Of course, there may be exceptional cases where the expertise and the requisite equipment may be expensive or scarce and specimens may still have to be exported. Sometimes exporting specimens may be cheaper than transporting an expert from the developed nation to work in the developing country.

Although there is always potential for exploitation in any international collaborative work, it is also important to consider the gains that may be shared. These include the possibility of earning income, academic growth, including publications and 
training, and direct improvement of care for patients in the developing nation. Patients can have haemoglobin, CD4 counts and other laboratory tests carried out, which would not have been carried out otherwise had there been no international collaboration. Most of the treatment resources for patients with Burkitt's lymphoma in Malawi have been through a collaborative research project.

\section{OWNERSHIP, CONTROL AND ACCESS}

The other issues to consider are aspects of ownership, control and access. Who owns the blood sample between the researcher and the research subject? Basically, no one really owns human body parts. The consideration that it is the research participant's blood is tempting, however. When blood or any human specimens are collected for research or clinical diagnostic purposes, control and access rest with the researcher or the laboratory. The subject may be accorded control or access to the specimen, but is usually not. In research where specimens are collected in one jurisdiction and transported to another, what is the access and control of the specimen between the IRB of the country sending the specimen and the IRB of the recipient country? If two research institutions are collaborating, one from a developing and another from a developed country, who should ultimately have control and access rights over the specimen? What are the rights and obligations of each of the researchers from both the developed and developing nations? These are issues necessitating international debate.

\section{DURATION OF STORAGE}

It is interesting to note that epidemiologically-useful information about HIV in Malawi was obtained from a human specimen stored for many years. Glynn $\mathrm{et}^{\mathrm{al}} \mathrm{l}^{7}$ in their studies in Karonga, Northern Malawi, reported that the earliest HIVpositive blood specimens were collected in 1982. At that time, the clinical syndrome that we now describe as AIDS had just been described in San Francisco in 1981 and in Malawi, AIDS was to be described later in 1985. Glynn et al's findings were only possible because they had the opportunity to analyse stored serum samples for an unrelated study. Glynn et al give valuable data, suggesting that HIV was present in Malawi earlier than had been realised. Seeff $e t a l^{8}$ have also studied the presence of hepatitis $\mathrm{C}$ antibodies in serum samples collected between 1948 and 1954, which at the time was aimed at studying group A streptococcal infection and acute rheumatic heart fever. ${ }^{9}$ The serum was frozen in rubber-capped glass vials at $20^{\circ} \mathrm{C}$. At the time of the study, hepatitis $\mathrm{C}$ had not yet been discovered. ${ }^{10}$

The storage of human specimens beyond the study for which the sample was collected, however, raises ethical concerns, even more so when the specimens may be used for studies that they were not intended for in the first place, in another country. Should research subjects fear or accept that their samples may be used for other things? Or should researchers clearly indicate to them that the sample could be used for other things, known or as yet unknown?

If human samples are to be used beyond the studies for which they were collected, what are the obligations of all the stakeholders-that is, researchers in all the collaborating institutions - towards each other, towards the research subjects and the community? Defining the "community" may also be problematic $^{11}$ as it could mean the research subjects cohort itself, the community from where they come from geographically or ethnically, or their families. As the institution from a developed nation usually stores the specimens, does it have the right to use the specimen without the collaboration of the researchers from the developing nation with whom they collaborated to collect the samples? What if useful information that has the potential to improve the health of the research subject is known? Should this new information be transmitted to the research subject when they had no knowledge in the first place that further analysis beyond the study had been done? If the researchers do not transmit back the new information obtained from the sample, are they not responsible for not helping in improving the health (beneficence) of research subjects?

These issues may be different if specimen collection is anonymised-that is, subjects are not required to submit their names. Coding of the identity of research participants and who has access to these codes depends on the particular design and on who has the power in the study. Varied experiences and varied power differentials exist within research projects, so that no blanket statement can be made. When subjects can be identified, it may be argued that it is the moral duty of the researchers to disclose any information that may be perceived as potentially useful to the research subjects.

We suggest that access and control of the specimens should rest with both research institutions, and not just the one storing the specimens. If one institution desires to transfer its right to access and control, this should be expressed in writing. We also recommend that IRBs attempt to know beforehand the plans that the collaborating institutions have regarding access and control of specimens in multi-institution studies. Providing access and control to both the collaborating institutions will ensure that the benefits arising from the use of the stored specimens are shared among the partner institutions. Exploitation of one by the other is therefore minimised.

\section{INFORMED CONSENT}

The researcher-subject relationship is based on trust, trust that the researcher has disclosed all material aspects of the study, especially potential harms and what the researcher intends to do with any specimens that may be collected. Informed consent implies that the researcher has given the information and that the subjects understand and can choose whether to participate. Although subjects may not choose which arm of the study they will be in, (eg, intervention or placebo), they can still choose not to participate in such trials if they are concerned about receiving placebos. Most researchers obtain consent from study subjects on the basis of the current study and not so much for a future, as yet unknown, study. In our setting here in Malawi, we suggest that it may be culturally unacceptable to keep specimens for many years and many research subjects may be uncomfortable with the prospect of having their samples stored for many years. Although we believe that storing human specimens for many years may not be culturally acceptable, we also subscribe to the notion that culture is dynamic and not "written in stone" and that given the right reasons for change, it can be changed.

Despite being advocated by bioethicists and researchers obtaining informed consent, like many other things in life, may not be perfect. Mc Cabe et al ${ }^{12}$ have reported that the process of obtaining informed consent among the Navago ethnic group of Canada resulted in "embarrassment, confusion, misperception that promoted mistrust". Obtaining consent for future use of specimens may also encourage mistrust by research participants and community towards the researchers. We suggest that anthropological research is conducted on the acceptability of communities towards storage of human samples for longer than the life of any current study.

\section{CONCLUSION}

The physical aspects of transport of human specimens from one national jurisdiction to the other are spelt out by the WHO, International Air Transport Association and other stakeholders. 
The aspects of control, access, duration of storage and use are less clear. It is necessary to develop a body of evidence and practice experience, which may guide rational and ethical collaboration between institutions. As Muthuswamy ${ }^{13}$ has suggested, collaboration between two institutions should ensure that the benefits of partnership go beyond people, and also beyond communities. We suggest that in multicountry research projects entailing the collection of human specimens, the following should be discussed and resolved before a study is conducted:

- Access and control of the specimens

- Which laboratory and other tests should be carried out locally and which ones abroad

- Future use of the specimens for research or at least a mechanism for discussion and resolution should the need arise

- Agreement on the distribution of benefits should the research result in such gains

We suggest that not considering these points is irresponsible and may also be construed as unethical.

\section{Authors' affiliations}

A S Muula, J M Mfutso-Bengo, Department of Community Health, University of Malawi, College of Medicine, Blantyre, Malawi J M Mfutso-Bengo, Malawi Bioethics Research Unit (MABIRU), Malawi

Competing interests: ASM is a Fogarty Infectious Diseases Fellow at the University of North Carolina at Chapel Hill. JMM-B is the Secretary of the
College of Medicine Research and Ethics Committee, the National Health Sciences Research Committee, and Data and Safety Monitoring Boards of the National Institutes of Health, USA.

\section{REFERENCES}

1 Ministry of Health and Population. Malawi fourth national health plan 19992004. Lilongwe, Malawi: Ministry of Health and Population, 1999.

2 Fenton PM, Whitty CJM, Reynolds F. Caesarian section in Malawi: prospective study of early maternal and perinatal mortality. BM J 2003;327:587.

3 Muula AS, Phiri A. Did maternal mortality ratio increase in Malawi between 1998-1998: review of Malawi Demographic and Health Survey and other data sources. Trop Doc 2003;33:182-5.

4 Muula AS. Ethical and programmatic challenges in antiretroviral scaling-up in Malawi: challenges in meeting the World Health Organization's "treating 3 million by 2005" initiative goals. Croat Med J 2004;45:415-21.

5 National Statistical Office (Malawi). Malawi Demographic and Health Survey 2000. Zomba, Malawi: National Statistical Office, 2001

6 Stirrat GM, Gill R. Autonomy in medical ethics after O'Niell. J Med Ethics 2005;31:127-30.

7 Glynn JR, Ponnighaus J, Crampin AC, et al. The development of the HIV epidemic in Karonga District, Malawi. AIDS 2001;15:2025-9.

8 Seeff LB, Miller RN, Rabkin CS, et al. 45-Year follow-up of hepatitis C virus infection in healthy young adults. Arch Intern Med 2000;132:105-11.

9 Denny FW, Wannamaker LW, Brink WR, et al. Prevention of rheumatic fever, treatment of preceding streptococcal infections in the United States Navy. JAMA 1950;143:151-3.

10 Choo QL, Kuo G, Weiner AJ, et al. Isolation of a c DNA clone derived from a blood borne non-A, non-B viral hepatist genome. Science 1989;244:359-62.

11 MacQueen KM, McLellan E, Metzger DS, et al. What is community? An evidence-based definition for participatory public health. Am J Public Health 2001:91:1929-38.

12 Mc Cabe M, Morgan F, Curley H, et al. The informed consent process in a crosscultural setting: is the process achieving the intended result? Ethn Dis 2005; 15:300-4.

13 Muthuswamy V. Ethical issues in HIV/AIDS research. Ind J Med Res 2005; 129:601-10

\section{BNF for Children 2006, second annual edition}

In a single resource:

- guidance on drug management of common childhood conditions

- hands-on information on prescribing, monitoring and administering medicines to children

- comprehensive guidance covering neonates to adolescents

For more information please go to bnfc.org 